\title{
A Family of Methods for Solving Nonlinear Equations with Twelfth-Order Convergence
}

\author{
Xilan Liu, Xiaorui Wang \\ Department of Mathematics and Statistics, Qinghai University for Nationalities, Xining, China \\ Email: doclanliu2002@yahoo.com.cn
}

Received August 17, 2012; revised January 11, 2013; accepted January 18, 2013

\begin{abstract}
This paper presents a new family of twelfth-order methods for solving simple roots of nonlinear equations which greatly improves the order of convergence and the computational efficiency of the Newton's method and some other known methods.
\end{abstract}

Keywords: Iterative Method; Nonlinear Equation; Twelfth-Order Convergence

\section{Introduction}

Solving nonlinear equations is one of the most important problems in numerical analysis. Generally, it is difficult to find the exact root of the nonlinear equations, and so iterative methods become the efficient way to obtain approximate solutions. Two important aspects related to iterative methods are order of convergence and computational efficiency. Order of convergence presents the speed at which a given iterative sequence converges to the root, and the computational efficiency shows the economy of the iterative scheme. In this paper, we will consider the above two aspects and establish a family of iterative methods to find the simple roots for the nonlinear equation $f(x)=0$, i.e., we will find $\alpha$ such that

$$
f(\alpha)=0, f^{\prime}(\alpha) \neq 0 .
$$

It is well known that the classical Newton's method is a basic and important iterative method [1] to find $\alpha$ by

$$
x_{n+1}=x_{n}-\frac{f\left(x_{n}\right)}{f^{\prime}\left(x_{n}\right)},
$$

which is quadratically convergent in the neighborhood of $\alpha$.

In recent years, many variants of accelerated Newton's methods have been proposed, for example [1-14]. In particular, $[1,14]$ constructed a variant of Newton's method via the iterative scheme:

$$
\left\{\begin{array}{l}
y_{n}=x_{n}-\frac{f\left(x_{n}\right)}{f^{\prime}\left(x_{n}\right)}, \\
x_{n+1}=x_{n}-\frac{2 f\left(x_{n}\right)}{f^{\prime}\left(x_{n}\right)+f^{\prime}\left(y_{n}\right)},
\end{array}\right.
$$

which converges cubically with three function evaluations per iteration and the computational efficiency index 1.442. In [10], the authors presented a new modification of Jarratt's method based on the circle of curvature which has the same convergent speed as our method.

Motivated by the recent activities in developing modified Newton's method, concerning both the order of convergence and the computational efficiency, we present a family of new iteration schemes for solving nonlinear equations with twelfth-order convergence which are better than Newton's method, the method provided by $[1,10,14]$, and can be used to find the simple roots of any type of nonlinear equation $f(x)=0$.

\section{Convergence Analysis}

Based on the iterative method provided by $[1,14]$, we construct the iterative scheme as follows:

$$
\left\{\begin{array}{l}
z_{n}=x_{n}-\frac{2 f\left(x_{n}\right)}{f^{\prime}\left(x_{n}\right)+f^{\prime}\left(y_{n}\right)}, \\
w_{n}=z_{n}-\frac{f\left(z_{n}\right)}{f^{\prime}\left(z_{n}\right)}, \\
x_{n+1}=w_{n}-\frac{f\left(z_{n}\right)+\beta f\left(w_{n}\right)}{f\left(z_{n}\right)+(\beta-2) f\left(w_{n}\right)} \frac{f\left(w_{n}\right)}{f^{\prime}\left(z_{n}\right)},
\end{array}\right.
$$

where $y_{n}=x_{n}-\frac{f\left(x_{n}\right)}{f^{\prime}\left(x_{n}\right)}$ and $\beta$ is an arbitrary real constant.

Theorem 2.1. Let $\alpha$ be a simple root of sufficiently smooth function $f: I \rightarrow R$ for an open interval $I$. If $x_{0}$ is sufficiently close to $\alpha$, then the method defined 
by (1) is at least twelfth-order, and its error equation is given by

$$
\begin{aligned}
e_{n+1}= & {\left[3 c_{2} c_{3}-(4+2 \beta) c_{2}^{3}\right]\left(c_{2}^{2}+\frac{1}{2} c_{3}\right)^{4} e_{n}^{12} } \\
& +O\left(e_{n}^{13}\right)
\end{aligned}
$$

where

$$
e_{n}=x_{n}-\alpha, c_{j}=(1 / j !) f^{(j)}(\alpha) / f^{\prime}(\alpha), j=1,2,3, \cdots .
$$

Proof: By Taylor expansion of the function $f(x)$ at point $\alpha$ and using the fact that $\alpha$ is a simple zero of $f(x)$, we have

$$
\begin{aligned}
& f\left(x_{n}\right) \\
& =f^{\prime}(\alpha)\left[e_{n}+c_{2} e_{n}^{2}+c_{3} e_{n}^{3}+c_{4} e_{n}^{4}+c_{5} e_{n}^{5}+c_{6} e_{n}^{6}+O\left(e_{n}^{7}\right)\right],
\end{aligned}
$$

and

$$
\begin{aligned}
& f^{\prime}\left(x_{n}\right) \\
& =f^{\prime}(\alpha)\left[1+2 c_{2} e_{n}+3 c_{3} e_{n}^{2}+4 c_{4} e_{n}^{3}+5 c_{5} e_{n}^{4}+6 c_{6} e_{n}^{5}+O\left(e_{n}^{6}\right)\right],
\end{aligned}
$$

Thus

$$
\begin{aligned}
\frac{f\left(x_{n}\right)}{f^{\prime}\left(x_{n}\right)} & =e_{n}-c_{2} e_{n}^{2}+2\left(c_{2}^{2}-c_{3}\right) e_{n}^{3} \\
& +\left(7 c_{2} c_{3}-4 c_{2}^{3}-3 c_{4}\right) e_{n}^{4} \\
& +\left(10 c_{2} c_{4}-20 c_{2}^{2} c_{3}-4 c_{5}+6 c_{3}^{2}+8 c_{2}^{4}\right) e_{n}^{5} \\
& +\left(13 c_{2} c_{5}-5 c_{6}-28 c_{2}^{2} c_{4}+17 c_{3} c_{4}\right. \\
& \left.+52 c_{2}^{3} c_{3}-33 c_{2} c_{3}^{2}+16 c_{2}^{5}\right) e_{n}^{6} \\
& +O\left(e_{n}^{7}\right)
\end{aligned}
$$

and

$$
\begin{aligned}
y_{n} & =\alpha+c_{2} e_{n}^{2}+2\left(c_{3}-c_{2}^{2}\right) e_{n}^{3} \\
& +\left(4 c_{2}^{3}+3 c_{4}-7 c_{2} c_{3}\right) e_{n}^{4} \\
& +\left(20 c_{2}^{2} c_{3}+4 c_{5}-10 c_{2} c_{4}-6 c_{3}^{2}-8 c_{2}^{4}\right) e_{n}^{5} \\
& +\left(5 c_{6}-13 c_{2} c_{5}+28 c_{2}^{2} c_{4}-17 c_{3} c_{4}\right. \\
& \left.-52 c_{2}^{3} c_{3}+33 c_{2} c_{3}^{2}-16 c_{2}^{5}\right) e_{n}^{6}+O\left(e_{n}^{7}\right) .
\end{aligned}
$$

Similarly, we have

$$
\begin{aligned}
f^{\prime}\left(y_{n}\right) & =f^{\prime}(\alpha)\left[1+2 c_{2}^{2} e_{n}^{2}+\left(4 c_{2} c_{3}-4 c_{2}^{3}\right) e_{n}^{3}\right. \\
& +\left(8 c_{2}^{4}+6 c_{2} c_{4}-11 c_{2}^{2} c_{3}\right) e_{n}^{4} \\
& +\left(28 c_{2}^{3} c_{3}+8 c_{2} c_{5}-20 c_{2}^{2} c_{4}-16 c_{2}^{5}\right) e_{n}^{5} \\
& +\left(10 c_{2} c_{6}-26 c_{2}^{2} c_{5}+60 c_{2}^{3} c_{4}-16 c_{2} c_{3} c_{4}\right. \\
& \left.\left.-68 c_{2}^{4} c_{3}-32 c_{2}^{6}\right) e_{n}^{6}+O\left(e_{n}^{7}\right)\right] .
\end{aligned}
$$

and

$$
\begin{aligned}
& \frac{2 f\left(x_{n}\right)}{f^{\prime}\left(x_{n}\right)+f^{\prime}\left(y_{n}\right)} \\
& =e_{n}-\left(c_{2}^{2}+\frac{1}{2} c_{3}\right) e_{n}^{3}+\left(3 c_{2}^{3}-\frac{3}{2} c_{2} c_{3}-c_{4}\right) e_{n}^{4} \\
& +\left(9 c_{2}^{2} c_{3}-6 c_{2}^{4}-2 c_{2} c_{4}-\frac{3}{2} c_{5}-\frac{3}{4} c_{3}^{2}\right) e_{n}^{5} \\
& +\left(15 c_{2}^{2} c_{4}-25 c_{2}^{3} c_{3}-\frac{5}{2} c_{2} c_{3}+8 c_{2}^{5}\right. \\
& \left.-2 c_{6}+4 c_{2} c_{3}^{2}+\frac{5}{2} c_{3} c_{4}\right) e_{n}^{6}+O\left(e_{n}^{7}\right) .
\end{aligned}
$$

Note that

$$
\begin{aligned}
z_{n} & =\alpha+\left(c_{2}^{2}+\frac{1}{2} c_{3}\right) e_{n}^{3}+\left(\frac{3}{2} c_{2} c_{3}-3 c_{2}^{3}+c_{4}\right) e_{n}^{4} \\
& +\left(6 c_{2}^{4}-9 c_{2}^{2} c_{3}+2 c_{2} c_{4}+\frac{3}{2} c_{5}+\frac{3}{4} c_{3}^{2}\right) e_{n}^{5} \\
& +\left(25 c_{2}^{3} c_{3}-15 c_{2}^{2} c_{4}+\frac{5}{2} c_{2} c_{3}-8 c_{2}^{5}\right. \\
& \left.+2 c_{6}-4 c_{2} c_{3}^{2}-\frac{5}{2} c_{3} c_{4}\right) e_{n}^{6}+O\left(e_{n}^{7}\right) .
\end{aligned}
$$

and using the Taylor expansions of $f\left(z_{n}\right)$ and $f^{\prime}\left(z_{n}\right)$, we have

$$
\begin{aligned}
& f\left(z_{n}\right) \\
= & f^{\prime}(\alpha)\left[\left(z_{n}-\alpha\right)+c_{2}\left(z_{n}-\alpha\right)^{2}+c_{3}\left(z_{n}-\alpha\right)^{3}\right. \\
& \left.+c_{4}\left(z_{n}-\alpha\right)^{4}+O\left(\left(z_{n}-\alpha\right)^{5}\right)\right],
\end{aligned}
$$

and

$$
\begin{aligned}
f^{\prime}\left(z_{n}\right)= & f^{\prime}(\alpha)\left[1+2 c_{2}\left(z_{n}-\alpha\right)+3 c_{3}\left(z_{n}-\alpha\right)^{2}\right. \\
& \left.+4 c_{4}\left(z_{n}-\alpha\right)^{3}+O\left(\left(z_{n}-\alpha\right)^{4}\right)\right] .
\end{aligned}
$$

Hence,

$$
\begin{aligned}
& w_{n} \\
& =z_{n}-\frac{f\left(z_{n}\right)}{f^{\prime}\left(z_{n}\right)} \\
& =\alpha+c_{2}\left(z_{n}-\alpha\right)^{2}+\left(2 c_{3}-2_{2}^{2}\right)\left(z_{n}-\alpha\right)^{3} \\
& \quad+\left(3 c_{4}+4 c_{2}^{3}-7 c_{2} c_{3}\right)\left(z_{n}-\alpha\right)^{4}+O\left(\left(z_{n}-\alpha\right)^{5}\right),
\end{aligned}
$$

and

$$
\begin{aligned}
& f\left(w_{n}\right) \\
& =f^{\prime}(\alpha)\left[c_{2}\left(z_{n}-\alpha\right)^{2}+\left(2 c_{3}-2_{2}^{2}\right)\left(z_{n}-\alpha\right)^{3}\right. \\
& \left.\quad+\left(3 c_{4}+6 c_{2}^{3}-7 c_{2} c_{3}\right)\left(z_{n}-\alpha\right)^{4}+O\left(\left(z_{n}-\alpha\right)^{5}\right)\right] .
\end{aligned}
$$


Furthermore, we can obtain that

$$
\begin{aligned}
& \frac{f\left(z_{n}\right)+\beta f\left(w_{n}\right)}{f\left(z_{n}\right)+(\beta-2) f\left(w_{n}\right)} \\
= & 1+2 c_{2}\left(z_{n}-\alpha\right)+2(\beta+1) c_{2}^{2}\left(z_{n}-\alpha\right)^{2} \\
& +\left[6 c_{4}+\left(4 \beta-2 \beta^{2}+2\right) c_{2}^{3}-(8+4 \beta) c_{2} c_{3}\right] \\
& \times\left(z_{n}-\alpha\right)^{3}+O\left(\left(z_{n}-\alpha\right)^{4}\right),
\end{aligned}
$$

and

$$
\begin{aligned}
& \frac{f\left(z_{n}\right)+\beta f\left(w_{n}\right)}{f\left(z_{n}\right)+(\beta-2) f\left(w_{n}\right)} \frac{f\left(w_{n}\right)}{f^{\prime}\left(z_{n}\right)} \\
= & c_{2}\left(z_{n}-\alpha\right)^{2}+\left(2 c_{3}-2 c_{2}^{2}\right)\left(z_{n}-\alpha\right)^{3} \\
& +\left[3 c_{4}+(6+2 \beta+2) c_{2}^{3}-10 c_{2} c_{3}\right] \\
& \times\left(z_{n}-\alpha\right)^{4}+O\left(\left(z_{n}-\alpha\right)^{5}\right) .
\end{aligned}
$$

it follows from (1) that

$$
\begin{aligned}
x_{n+1}= & \alpha+\left[3 c_{2} c_{3}-(4+2 \beta) c_{2}^{3}\right]\left(z_{n}-\alpha\right)^{4} \\
& +O\left(\left(z_{n}-\alpha\right)^{5}\right),
\end{aligned}
$$

that is

$$
e_{n+1}=\left[3 c_{2} c_{3}-(4+2 \beta) c_{2}^{3}\right]\left(c_{2}^{2}+\frac{1}{2} c_{3}\right)^{4} e_{n}^{12}+O\left(e_{n}^{13}\right) .
$$

The proof is complete.

\section{Numerical Examples}

We give some examples to illustrate the efficiency of the new iterative method (1) with $\beta=2$ (denoted by NVNM) and compare the method with Newton's method (NM), the method provided by [1] (VNM), [10] (YM) and [14] (VNM).

We use the following stopping criteria for computer programs:

$$
\left|x_{n+1}-x_{n}\right|<\sqrt{\varepsilon} \text { and }\left|f\left(x_{n+1}\right)\right|<\sqrt{\varepsilon},
$$

where $\varepsilon=10^{-28}$, which are the same as those used in [14].

The test functions are listed as follows:

$$
\begin{aligned}
& f_{1}(x)=x^{3}+4 x^{2}-10, f_{2}(x)=\sin ^{2} x-x^{2}+1, \\
& f_{3}(x)=\mathrm{e}^{x}+x-20, f_{4}(x)=\cos x-x, \\
& f_{5}(x)=(x-1)^{3}-1, f_{6}(x)=x^{3}-10, \\
& f_{7}(x)=x \mathrm{e}^{x^{2}}-\sin ^{2} x+3 \cos x+5, \\
& f_{8}(x)=\mathrm{e}^{x^{2}+7 x-30}-1 .
\end{aligned}
$$

\begin{tabular}{|c|c|c|c|c|c|c|c|c|c|c|}
\hline \multirow[t]{2}{*}{$f(x)$} & \multirow[t]{2}{*}{$x_{0}$} & \multicolumn{4}{|c|}{$\mathrm{N}$} & \multicolumn{4}{|c|}{ NOFE } & \multirow[t]{2}{*}{ ROOT } \\
\hline & & NM & VNM & YM & NVNM & NM & VNM & YM & NVNM & \\
\hline \multirow{2}{*}{$f_{1}(x)$} & -0.5 & 112 & 6 & 28 & 3 & 224 & 18 & 168 & 18 & \multirow{2}{*}{$\alpha=1.36523001341410$} \\
\hline & -0.3 & 53 & 6 & 12 & 6 & 106 & 18 & 72 & 36 & \\
\hline \multirow{2}{*}{$f_{2}(x)$} & 1 & 6 & 6 & 2 & 2 & 12 & 18 & 12 & 12 & \multirow{2}{*}{$\alpha=1.40449164821534$} \\
\hline & 3 & 6 & 3 & 2 & 2 & 12 & 9 & 12 & 12 & \\
\hline \multirow{2}{*}{$f_{3}(x)$} & 2 & 6 & 4 & 2 & 2 & 12 & 12 & 12 & 12 & \multirow{2}{*}{$\alpha=0.25753038543986$} \\
\hline & 1.5 & 7 & 5 & 2 & 3 & 14 & 15 & 12 & 18 & \\
\hline \multirow{2}{*}{$f_{4}(x)$} & 1 & 4 & 2 & 2 & 1 & 8 & 6 & 12 & 6 & \multirow{2}{*}{$\alpha=0.73908513321516$} \\
\hline & 0.5 & 4 & 3 & 2 & 1 & 8 & 9 & 12 & 6 & \\
\hline \multirow{2}{*}{$f_{5}(x)$} & 3.5 & 7 & 5 & 2 & 2 & 12 & 15 & 12 & 12 & \multirow{2}{*}{$\alpha=2$} \\
\hline & 2.5 & 6 & 4 & 2 & 2 & 12 & 12 & 12 & 12 & \\
\hline$f_{6}(x)$ & 1.5 & 6 & 4 & 2 & 2 & 12 & 12 & 12 & 12 & $\alpha=2.15443469003188$ \\
\hline$f_{7}(x)$ & -2 & 8 & 6 & 3 & 3 & 16 & 18 & 18 & 18 & $\alpha=-1.20764782713092$ \\
\hline$f_{8}(x)$ & 3.5 & 12 & 8 & 4 & 6 & 24 & 24 & 24 & 36 & $\alpha=3$ \\
\hline
\end{tabular}

The computational results in the Table 1 show that the method NVNM requires less NOFE than NM, and less NOFE than VNM in most cases. So, it is better in practical interest.

\section{Conclusion}

It is shown that the method (1) converges to the root. The computational efficiency index is 1.513 which is bigger than the index of NM 1.414 and the index of VNM 1.442. The method constructed in this paper is more efficient and performs better than classical Newton's method and the method presented by $[1,10,14]$.

Table 1. Comparison of some iterative methods.

$\mathrm{N}$ : Numbers of iterations; NOFE: Numbers of function evaluations. 


\section{Acknowledgements}

This paper was supported by the NNSF (10961020), the Chunhui Project of Chinese Ministry of Education (Z2009-1-81007), the 135 Talent Project of Qinghai Province and the Foundation of the Chinese Nationality Committee (10QH03).

\section{REFERENCES}

[1] J. F. Traub, "Iterative Methods for Solutions of Equations," Prentice-Hall, Upper Saddle River, 1964.

[2] C. Chun, "Some Third-Order Families of Iterative Methods for Solving Nonlinear Equations," Applied Mathematics and Computation, Vol. 188, No. 1, 2007, pp. 924933. doi:10.1016/j.amc.2006.09.113

[3] C. Chun and Y. M. Ham, "Some Second-Derivative-Free of Super-Halley Method with Fourth-Order Convergence," Applied Mathematics and Computation, Vol. 195, No. 2, 2008, pp. 532-541. doi:10.1016/j.amc.2007.05.003

[4] M. T. Darvish and A. Barati, "A Third-Order NewtonType Method to Solve Systems of Nonlinear Equations," Applied Mathematics and Computation, Vol. 187, No. 2, 2007, pp. 630-635. doi:10.1016/j.amc.2006.08.080

[5] M. T. Darvish, "A Two-Step High-Order Newton-Like Method to Solve Systems of Nonlinear Equations," International Journal of Pure and Applied Mathematics, Vol. 57, No. 4, 2009, pp. 543-555.

[6] M. T. Darvishi, "Some Three-Step Iterative Methods Free from Second Order Derivative for Finding Solutions of Systems of Nonlinear Equations," International Journal of Pure and Applied Mathematics, Vol. 57, No. 4, 2009, pp. 557-573.

[7] M. Frontini and E. Sormani, "Third-Order Methods from
Quadrature Formulae for Solving Systems of Nonlinear Equations," Applied Mathematics and Computation, Vol. 149, No. 3, 2004, pp. 771-782. doi:10.1016/S0096-3003(03)00178-4

[8] H. H. H. Homeier, "On Newton-Type Methods for Multiple Roots with Cubic Convergence," Journal of Computational and Applied Mathematics, Vol. 231, No. 1, 2009, pp. 249-254. doi:10.1016/j.cam.2009.02.006

[9] J. Kou, Y. Li and X. Wang, "An Improvement of the Jarratt Method," Applied Mathematics and Computation, Vol. 189, No. 2, 2007, pp. 1816-1821. doi:10.1016/j.amc.2006.12.062

[10] Y.-I. Kim and C. Chun, "New Twelfth-Order Modifications of Jarratt's Method for Solving Nonlinear Equations," Studies in Nonlinear Sciences, Vol. 1, No. 1, 2010, pp. 14-18.

[11] F. Liang, G. P. He and Z. Y. Hu, "A Cubically Convergent Newton-Type Method under Weak Conditions," Journal of Computational and Applied Mathematics, Vol. 220, No. 1-2, 2008, pp. 409-412. doi:10.1016/j.cam.2007.08.013

[12] M. A. Noor and K. I. Noor, "Modified Iterative Methods with Cubic Convergence for Solving Nonlinear Equations," Applied Mathematics and Computation, Vol. 184, No. 2, 2007, pp. 322-325. doi:10.1016/j.amc.2006.05.155

[13] J. R. Sharma, R. K. Guha and R. Sharma, "Improved Ostrowski-Like Methods Based on Cubic Curve Interpolation," Applied Mathematics, Vol. 2, No. 7, 2011, pp. 816-823. doi:10.4236/am.2011.27109

[14] S. Weerakoon and T. G. I. Fernando, "A Variant of Newton's Method with Accelerated Third-Order Convergence," Applied Mathematics Letter, Vol. 13, No. 8, 2000, pp. 87-93. doi:10.1016/S0893-9659(00)00100-2 